\title{
Is there a tower of charges to be discovered?
}

\author{
T Månsson \\ Max-Planck Institut für Gravitationsphysik, Albert-Einstein-Institut Am Mühlenberg 1, \\ D-14476 Potsdam, Germany \\ E-mail: teresia@aei.mpg.de
}

Received 31 October 2007, in final form 28 January 2008

Published 29 April 2008

Online at stacks.iop.org/JPhysA/41/194014

\begin{abstract}
We investigate higher-loop integrability for a $q$-deformation of the $\mathfrak{s u}(2)$-sector of $\mathcal{N}=4 \mathrm{SYM}$ theory. First we construct a generalization of the longrange spin chain, which for the lowest orders describes the non-deformed dilatation operator. This generalized model is built up from TemperleyLieb algebra generators and describes the deformed theory to at least two loops. When constructing the model we have demanded the existence of one commuting charge, which puts strong constraints on the parameters to threeloop orders. We also write the five first charges for this model at two-loops order. Our main goal is to obtain an explicit expression for an infinite number of commuting charges, all commuting with the dilatation operator. This would imply integrability. As a step towards this goal we present in this paper an expression for a generic local charge of the one-loop dilatation operator, which happens to be a generator of the Temperley-Lieb algebra.
\end{abstract}

PACS numbers: $02.30 . \mathrm{Ik}, 11.25 . \mathrm{Tq}, 75.10 . \mathrm{Pq}$

(Some figures in this article are in colour only in the electronic version)

\section{Introduction}

One decade ago, Maldacena conjectured the famous AdS/CFT correspondence relating a strongly coupled conformal field theory (in particular the so-called $\mathcal{N}=4$ supersymmetric Yang-Mills theory) with a weakly coupled string theory on an Anti-de Sitter background and vice versa. The energies of the strings are mapped to the anomalous dimensions of certain single trace operators (composite gauge invariant operators) in the field theory. If the duality was correct it would enable us to calculate quantities in either theory which were previously almost unattainable.

Integrability has shown up in a remarkable way in the context of $\mathcal{N}=4$ supersymmetric Yang-Mills theory with the gauge group $S U\left(N_{c}\right)$, when calculating the anomalous dimensions of single trace operators in the large $N_{c}$-limit (the planar limit). In particular, it has been 
pointed out that the dilatation operator, which is defined to give the anomalous dimensions as its eigenvalues, can, to one-loop order, be mapped to an integrable spin chain [1, 2]. Furthermore, in some sectors in the planar limit of the theory, the dilatation operator has been proven to be integrable to a few-loops order. In particular, for the $\mathfrak{s u}(2)$ sector the dilatation operator is now known to four-orders accuracy [3-5]. The exact expression for the operator is not yet clear. For the $\mathfrak{s u}(2)$ sector it has been shown [6] that the first few orders agree with the Inozemtsev spin chain [7], and it can also be obtained as a limiting case of the BDS-Hubbard model [8]. It is believed that the theory is integrable to all-loops order in all sectors. A conjectured all-loop asymptotic Bethe ansatz has been written [9-11].

Deformations of the theory are of physical interest. Integrability has been studied for some deformations preserving the conformal structure of the theory, in particular the $q$-deformation (or Leigh-Strassler deformation) [12-17]. There it has been found that the one-loop dilatation operator in the planar limit is integrable for specific values of the parameters and in particular sectors, but beyond one loop not much is known [18].

It is an interesting question if there exists a natural generalization of the integrable all-loop model for the $\mathfrak{s u}(2)$ case, to the $q$-deformed $\mathfrak{s u}(2)$-sector. By $q$-deformed, we now mean the $q$-deformed Leigh-Strassler deformation of $\mathcal{N}=4$ supersymmetric Yang-Mills theory [19]. In practice, that means that the operator $I-P$ should be exchanged with the Temperley-Lieb generators $e_{i}$ in the Feynman diagrams which give rise to the dilatation operator. These types of generalizations appear to have been fruitful before, e.g. the model discussed in [20].

The spin chain Hamiltonian, to which the dilatation operator is mapped, can in the nondeformed $\mathcal{N}=4 \mathrm{SYM}$ case be written as a loop expansion $[3,6]$,

$$
H=L+\sum_{k=1}^{\infty}\left(\frac{\lambda}{16 \pi^{2}}\right)^{k} h_{k},
$$

where the first three $h_{k}$ are

$$
\begin{aligned}
& h_{2}=-6 L+8 \sum_{i} P_{i, i+1}-2 \sum_{i}\left\{P_{i, i+1}, P_{i+1, i+2}\right\} \\
& h_{3}=60 L+\sum_{i}-104 P_{i, i+1}+24\left\{P_{i, i+1}, P_{i+1, i+2}\right\}+4 P_{i, i+1} P_{i+2, i+3} \\
& \quad-4\left(P_{i, i+1} P_{i+1, i+2} P_{i+2, i+3}-P_{i+2, i+3} P_{i+1, i+2} P_{i, i+1}\right),
\end{aligned}
$$

where $L$ is the length of the trace operator in the field theory, or alternatively the length of the spin chain.

The lowest known orders of the dilatation operator for the non-deformed theory satisfy perturbative integrability ( $K$ is the number of known orders):

$$
Q_{l}=\sum_{k=0}^{K} \lambda^{k} Q_{l}^{(k)}, \quad\left[Q_{l}, Q_{m}\right]=\mathcal{O}\left(\lambda^{(K+1)}\right)
$$

Up to the first few orders, the Hamiltonian agrees with an effective spin chain model obtained as a certain limit of the Hubbard model. We would like to see if this is also the case for our generalization of the model.

We have two main motivations for this paper, one is to clarify whether the $q$-deformed $\mathfrak{s u}(2)$ model can be integrable to higher loops, and secondly on a very general basis investigate the possibility to generalize the type of long-range integrable spin chain describing the dilatation operator in the $\mathfrak{s u}(2)$ sector in $N=4 \mathrm{SYM}$ theory, to integrable long-range systems built up with Temperley-Lieb generators. We will start by writing the simplest long-range model to four orders in $\lambda$ which to three-loops order reduces in the $q \rightarrow 1$ limit to the one 
above, modulo commuting charges. We would like to show that this generalized model is indeed integrable. Our plan is to prove the existence of an infinite number of commuting charges, by writing an explicit expression for them. In order to do so we would first need to have a generic expression for any local charge commuting with the first order charge. The first-order charge can be represented by a generator $e_{l}$ of the Temperley-Lieb algebra.

In $[21,22]$, the authors derived expressions for a generic charge of the Heisenberg spin chain. In [23], it was shown that the Hubbard model does not have a boost operator. This makes it harder to derive the general charge. One motivation for them to understand the structure of the charges for the Heisenberg spin chain, was that it could reveal the structure of the charges of the Hubbard model, and also of long-range spin chains which has the Heisenberg spin chain as a limiting case, i.e. the Haldane-Shastry spin chain.

We think that the underlying structure of the charges at one loop will be related in the same way to a deformed Hubbard model or Haldane-Shastry model. According to our knowledge, very little is known about long-range integrable models and we expect this generalization to shed some light into this area.

\section{The $q$-deformed $\mathfrak{s u}(2)$ sector}

In the case of the $q$-deformed $\mathfrak{s u}(2)$ sector, to one-loop accuracy, the action of the dilatation operator on two adjacent scalar fields in the trace operator can be described by either the action of the XXZ spin-1/2 chain (when considering the sector with one holomorphic and one anti-holomorphic scalar field [17]), or a 2D representation of the Temperley-Lieb algebra (when considering the gauge theory sector with two holomorphic, or two anti-holomorphic scalar fields $[12,17])$. The action of $e_{i}$ on two neighbouring spin sites $i$ and $i+1$, in the latter case, can be can be represented by the matrix

$$
e_{i}=\left(\begin{array}{cccc}
0 & 0 & 0 & 0 \\
0 & |q| & -\mathrm{e}^{\mathrm{i} \beta} & 0 \\
0 & -\mathrm{e}^{-\mathrm{i} \beta} & |q| & 0 \\
0 & 0 & 0 & 0
\end{array}\right),
$$

where $q=|q| \mathrm{e}^{\mathrm{i} \beta}(\beta \in \mathbb{R})$ is the deformation parameter, which deforms the superpotential in the field theory.

In the spin chain description, we consider closed chains since these correspond to trace operators in the field theory. The difference between the XXZ spin chain Hamiltonian and the Temperley-Lieb generator vanishes in the case of closed spin chains. Thus we write the one-loop dilatation operator as a sum of generators of the Temperley-Lieb algebra,

$$
Q_{1}=\sum_{l=1}^{L} e_{l},
$$

where $L$ is the length of the spin chain, which is periodic. The generators $e_{i}$ act on two adjacent spin sites $i$ and $i+1$, and fulfil the algebraic relations

$$
\begin{array}{ll}
e_{i} e_{i \pm 1} e_{i}=e_{i} & \\
e_{i} e_{j}=e_{j} e_{i}, & |i-j| \geqslant 2 \\
e_{i}^{2}=\gamma e_{i}, & \gamma=|q|+|q|^{-1} .
\end{array}
$$

We should point out that even though we are looking at this system with particular interest in the representation of the deformed $\mathfrak{s u}(2)$-sector, everything that follows is much 
more general, and is independent of the choice of representation. Only the fundamental Temperley-Lieb algebra above is used in the sequel.

Now we would like to start discussing what is our real interest, the higher-loop contributions to the dilatation operator. Up to one loop it is clear that due to the periodicity it does not matter which two scalar fields we choose as our representation. At higher-loop order we get contributions consisting of products of these main building blocks, and in principle we should check whether periodicity implies that the difference term vanishes also for these products.

For every increasing loop order, we have one more site involved in the interaction, as can be seen from the structure of the Feynman diagrams and the fact that we are taking the planar limit. At one-loop order, there are only nearest neighbour interactions, at two loops there are three adjacent spin sites involved and so on. In addition, the interaction can be expressed in terms of products of the generators $e_{i}$, and they always come in pairs with another product with the ordering reversed, i.e. the interaction operator $e_{1} e_{4} e_{3} e_{2}$ will be accompanied by the operator $e_{2} e_{3} e_{4} e_{1}$, with the same coefficient in front.

The most general expression satisfying these conditions to three loops, modulo commuting charges and linear combinations of the charge itself multiplied by constants times $\lambda$, is

$$
\begin{array}{r}
Q_{1}=\lambda\left[e_{i}+\lambda\left\{e_{i}, e_{i+1}\right\}+\lambda^{2}\left(\alpha_{1}\left\{e_{i}\left\{e_{i+1}, e_{i+2}\right\}\right\}+\alpha_{2}\left\{e_{i}, e_{i+2}\right\}\right)\right. \\
\left.\times \lambda^{3}\left(\left\{e_{i}\left\{e_{i+1},\left\{e_{i+2}, e_{i+3}\right\}\right\}\right\}+\cdots\right)\right]+\mathcal{O}\left(\lambda^{5}\right) .
\end{array}
$$

Our four-loop term is not the most general possibility. At four loops order we will at the moment not take interest in the most general expression. We will, however, use a form with maximum interaction range, and include the total anti-symmetrization of the four adjacent spin sites.

Our basic question now is whether this type of model is integrable, and for which parameter values. All we know from the outset is that it is integrable when $q \rightarrow 1$. Thus we know which parameter values are needed in that limit. A condition for integrability is that there must exist a $Q_{2}^{(2)}$ such that

$$
\left[Q_{1}^{(1)}, Q_{2}^{(2)}\right]=\left[Q_{2}^{(2)}, Q_{1}^{(1)}\right],
$$

assuming that the charge number $k$ is perturbatively expressed as

$$
Q_{k}=\sum_{\lambda=1}^{\infty} Q_{k}^{(l)} \lambda^{l}
$$

Condition (6) comes from demanding perturbative integrability. Before going on to write the charges that fulfil this condition, let us introduce some useful notation.

All expressions we will deal with will be combinations of commutators and anticommutators of the Temperley-Lieb generators $e_{i}$. Therefore, it will be convenient for us to introduce the following notation,

$$
e_{i_{1}, i_{2}, \bar{z}_{3}, i_{4}, \bar{i}_{5}, i_{6}}=\left[e_{i_{1}}\left[e_{i_{2}}\left\{e_{i_{3}}\left[e_{i+4}\left\{e_{i_{5}}, e_{i_{6}}\right\}\right]\right\}\right]\right] \text {, }
$$

which naturally reduces to a Temperley-Lieb generator when there is only one index. We also like to define a special 'index' $B_{i}$, which we define to mean

$$
e_{B_{i}}:=e_{i} e_{i-1} e_{i+1} e_{i} .
$$

We would like to introduce some more notation for future convenience. We will encounter terms of the form

$$
e_{\tilde{i}_{1}, \tilde{i}_{2}, \ldots, \tilde{i}_{k}, \ldots, \tilde{i}_{l}}, \quad \text { where } \quad i_{k}<i_{k+1},
$$


where the tilde means that the index can be either barred or not. It is practical to have a special word for the number $i_{l}-i_{1}+1$, where $i_{1}$ is the lowest index $i_{1}$ of the term and $i_{l}$ is the highest index. Let us call it 'range number'. We also need a special word for the total number of gaps. If, e.g., $i_{k}=i_{k+1}+2$, that would be a gap of one. The total number of gaps is the sum of all $g_{k}$, where $i_{k}=i_{k+1}+1+g_{k}$. We can characterize the terms by their range number, number of bars (anti-commutators), and total number of gaps.

We now introduce the notation

$$
T_{k, j}^{N}
$$

which will be a sum of terms with range number $N$, number of bars $k$ and total gap number $j$. As an example,

$$
\begin{aligned}
T_{2,1}^{5}=\sum e_{\overline{i+1}}, \overline{i+3}, i+4, i+5 & +e_{\overline{i+1}, i+3, \overline{i+4}, i+5}+e_{\overline{i+1}, \overline{i+2}, i+4, i+5} \\
& +e_{i+1, \overline{i+2}, \overline{i+4}, i+5}+e_{\overline{i+1}, i+2, \overline{i+3}, i+5}+e_{i+1, \overline{i+2}, \overline{i+3}, i+5} .
\end{aligned}
$$

We also introduce the notation $T B_{l, k}^{N}$, which is the same as $T_{l, k}^{N}$ but with one of the indices being the symbol $B_{i}$, e.g.,

$$
T B_{1,0}^{5}=\sum e_{\bar{B}_{i+1}, i+3, i+4}+e_{B_{i+1}, \overline{i+3}, i+4}+e_{\bar{i}, B_{i+2}, i+4}+e_{i, \bar{B}_{i+1}, i+3}+e_{\bar{i}, i+1, B_{i+3}}+e_{i, \bar{i}+1, B_{i+3}} .
$$

Now we are ready to use our notation to write two charges fulfilling restriction (6). After demanding the existence of one commuting charge, the coefficients $\alpha_{1}$ and $\alpha_{2}$ are completely determined. To fourth order we have several options, and here we have just chosen one possibility

$$
\begin{array}{r}
Q_{1}=T_{0,0}^{1}+\lambda T_{1,0}^{2}+\lambda^{2}\left(-\frac{2}{\gamma} T_{1,1}^{3}+T_{2,0}^{3}\right)+\lambda^{3}\left(\left(-1+\frac{2}{\gamma}-\frac{2}{\gamma^{2}}\right) T_{1,1}^{3}\right. \\
\left.-\frac{2}{\gamma} T_{2,0}^{3}+\left(\frac{2}{\gamma}-\frac{10}{\gamma^{2}}\right) T_{1,2}^{4}-\frac{2}{\gamma} T_{2,1}^{4}+2 T B_{0,0}^{3}+T_{3,0}^{4}\right),
\end{array}
$$

and the second charge

$$
\begin{aligned}
Q_{2}=T_{00}^{2}+\lambda & T_{1,0}^{3}+\lambda^{2}\left(-\frac{2}{\gamma}\left(T_{1,1}^{4}+T_{1,0}^{3}\right)+T_{2,0}^{4}\right)+\lambda^{3}\left(-\left(1-\frac{4}{\gamma}+\frac{12}{\gamma^{2}}\right) T_{1,1}^{4}\right. \\
& \left.-\left(4-\frac{2}{\gamma}+\frac{2}{\gamma^{2}}\right) T_{1,0}^{3}+\left(\frac{2}{\gamma}-\frac{10}{\gamma^{2}}\right) T_{1,2}^{5}-\frac{2}{\gamma}\left(T_{2,1}^{5}+2 T_{2,0}^{4}\right)+2 T B_{0,0}^{4}+T_{3,0}^{5}\right) .
\end{aligned}
$$

From this little exercise we can conclude that to second-loop order we are totally free, and the parameter values we would get from a field theory calculation do not matter. But to third order they are essential. Thus, by doing a three-loop field theory calculation we could exclude integrability if the coefficients do not come out as above. We also see that we have a $q$-dependence in the coefficients. It would be quite amazing if the field-theoretical calculation turned out to give exactly this dependence.

In cases where a boost operator that generates all the charges exists, it is enough to show that the first and second charges commute. It then follows that all the rest will commute. For the one-loop Hamiltonian, the boost operator is well known [24],

$$
B=\sum_{l=-\infty}^{\infty} l e_{l}
$$

Note that the boost operator is only well defined for the infinite spin chain, but this is not a problem. Unfortunately, when trying to find any boost operator for the higher-loop dilatation 
operator, we run into the same problem as in the case of the Hubbard model with a factor of two appearing, which destroys the ansatz for the boost operator from working [23].

In order to extract the generic charge commuting with our Hamiltonian, we first need an explicit expression for the charges in the one-loop case. In the following section we will derive a simple expression for a generic local charge of the Hamiltonian (3).

\section{The infinite tower}

The fact that the charges can be generated using the boost operator (14) gives us information about their algebraic structure. We claim that they can be expressed purely in terms of the quantities $T_{k, l}^{N}$ defined in the previous section.

We will now provide an induction proof for this claim. As seen in the previous section, this is already true for the lowest charges. We now prove it for the charge $Q_{N+1}$, assuming that it is true for $Q_{N}$. Due to the existence of the boost operator (14), the set of charges can be generated recursively as follows:

$$
Q_{N+1}=\left[B, Q_{N}\right] .
$$

Now we consider a generic term in the $Q_{N}$ charge, and study the result of commutation with the boost operator. The following relations are almost all we need to know in order to calculate commutators. First the relations involving one anti-commutator, i.e. one barred index:

$$
\begin{aligned}
& e_{2,1, \overline{2}, 3}=e_{\overline{2}, 3}-e_{\overline{1}, 2}-\frac{\gamma}{2} e_{\overline{1}, \overline{2}, 3}+\frac{\gamma}{2} e_{1,2,3}+2 e_{B_{2}} \\
& e_{2, \overline{1}, 2,3}=e_{\overline{2}, 3}-e_{\overline{1}, 2}+\frac{\gamma}{2} e_{\overline{1}, \overline{2}, 3}-\frac{\gamma}{2} e_{1,2,3}-2 e_{B_{2}} \\
& e_{\overline{2}, 1,2,3}=e_{\overline{2}, 3}+e_{\overline{1}, 2}-\frac{\gamma}{2} e_{\overline{1}, \overline{2}, 3}+\frac{\gamma}{2} e_{1,2,3}-2 e_{B_{2}} .
\end{aligned}
$$

Then the relations involving even number of anti-commutators:

$$
\begin{aligned}
& e_{2,1,2,3}=e_{2,3}-e_{1,2}-\frac{\gamma}{2} e_{\overline{1}, 2,3}+\frac{\gamma}{2} e_{1 \overline{2}, 3} \\
& e_{2, \overline{1}, \overline{2}, 3}=e_{2,3}-e_{1,2}+\frac{\gamma}{2} e_{\overline{1}, 2,3}-\frac{\gamma}{2} e_{1, \overline{2}, 3} \\
& e_{\overline{2}, \overline{1}, 2,3}=e_{2,3}+e_{1,2}+\frac{\gamma}{2} e_{\overline{1}, 2,3}-\frac{\gamma}{2} e_{1, \overline{2}, 3} \\
& e_{\overline{2}, 1, \overline{2}, 3}=e_{2,3}+e_{1,2}-\frac{\gamma}{2} e_{\overline{1}, 2,3}+\frac{\gamma}{2} e_{1, \overline{2}, 3},
\end{aligned}
$$

and lastly, the two simple relations:

$$
\begin{aligned}
& e_{1,1,2}=\gamma e_{\overline{1}, 2}-2 e_{1} \\
& e_{1, \overline{1}, 2}=\gamma e_{1,2} .
\end{aligned}
$$

The above relations are sufficient for our calculations, because all terms $e_{i_{1} i_{2} \ldots i_{k} \ldots i_{l}}$ in $T_{k, l}^{N}$ have index values ordered as $i_{k}<i_{k+1}$. When commuting with an element $e_{k}$ there will be at most four index values involved, as can be seen from the Temperley-Lieb algebra (4), because $e_{k}$ will commute with all generators $e_{i}$ appearing in $e_{1, \ldots, k-1, k, k+1, \ldots, N}$, except $e_{k-1}$ and $e_{k+1}$. When we commute $e_{k}$ with a generic term $e_{1, \ldots, \widetilde{k-1}, \tilde{k}, \widetilde{k+1}, \ldots, N}(\tilde{k}$ denotes $k$ or $\bar{k})$, we get

$$
\left[e_{k}, e_{1, \ldots, \widetilde{k-1}, \widetilde{k}, \widetilde{k+1}, \ldots, N}\right]=e_{1, \ldots, k, \widetilde{k-1}, \widetilde{k}, \widetilde{k+1}, \ldots, N} .
$$


In each term of the quantity $T_{k, l}^{N}$, each generator $e_{i}$ appears at most once. Looking at equations (16)-(18) and (19)-(22), the first set of equations give rise to terms for which this is not the case, because these contain the term $e_{B_{2}}$. It is not possible to rewrite it so that each generator appears at most once.

Thus, when commuting $Q_{N}$ with the boost operator, the only combinations of barred indices which can give rise to an undesirable term are

$$
\begin{aligned}
& {\left[n e_{n}, e_{1, \ldots, \overline{n-1}, n, n+1, \ldots]}=n e_{1, \ldots, n, \overline{n-1}, n, n+1, \ldots} .\right.} \\
& {\left[n e_{n}, e_{1, \ldots, n-1, \bar{n}, n+1, \ldots]}=n e_{1, \ldots, n, n-1, \bar{n}, n+1, \ldots .} .\right.}
\end{aligned}
$$

These two terms in $Q_{N}$ belong to the same $T_{k, l}^{N}$, which means they have the same prefactor. From the relative minus signs in equations (16)-(17), we see that the $e_{B_{2}}$ terms cancel when adding the two quantities on the right-hand sides of (25)-(26). In order for the sum above not to vanish we need to have an anti-commutator either connecting to the right (a bar over $n+1$ ) or the left (a bar over the index left of $n$, say it is $n-2$ ). Assume that it is to the left. Then the sum of the two terms above is

$$
2 n e_{1, \ldots, \overline{n-2}, \bar{n}, n+1, \ldots} .
$$

We can also obtain this type of term from the commutators

$$
\begin{aligned}
{\left[(n-2) e_{n-2},\right.} & \left.e_{1, \ldots, n-3, n-2, \overline{n-1}, \bar{n}, \ldots}\right]=(n-2) e_{1, \ldots, n-2, n-3, n-2, \overline{n-1}, \bar{n}, \ldots} \\
& -\frac{2}{\gamma}\left[n e_{n}, e_{1, \ldots, \overline{n-2}, n, n+1, \ldots]}\right]=-\frac{2}{\gamma} n e_{1, \ldots, \overline{n-2}, n, n, n+1, \ldots,}, \\
& -\frac{2}{\gamma}\left[(n+1) e_{n+1}, e_{1, \ldots, \overline{n-2}, n, n+1, \ldots}\right]=-\frac{2}{\gamma}(n+1) e_{1, \ldots, \overline{n-2}, n+1, n, n+1, \ldots} .
\end{aligned}
$$

The factor $2 / \gamma$ in the product with one gap and one bar is important in order for the $n$ to cancel, and it will be of importance for the appearance of the general charge (see equation (32)). Once again, using the relations (16)-(18), we add the three terms above and get (among other things)

$$
-(2 n-3) e_{1, \ldots, \overline{n-2}, \bar{n}, n+1, \ldots} \text {. }
$$

Thus we will get both the contributions (27) and (31). Adding them up, it is clear that we get these terms symmetrically distributed with no $n$-dependence. In this way we see that we obtain the correct structure. We also know from the existence of the boost operator that the term with the highest range number is $e_{1,2,3, \ldots, N}$.

Now we do the last preparation for what is needed to obtain the exact form of the commuting charges. First, we introduce the notation

$$
\tilde{T}_{k}^{N}=\sum_{j=0}^{k-1}\left(-\frac{\gamma^{-j}}{2}\right) T_{k-j, j}^{N}
$$

After some lengthy calculation, we can show that the following relation holds, valid when $1<k<N-4$ :

$$
0 \stackrel{N-1}{=}\left[\tilde{T}_{0}^{1}, \tilde{T}_{k}^{N}+\frac{\gamma}{2}\left(\tilde{T}_{k+1}^{N-1}-\tilde{T}_{k-1}^{N-1}\right)+\frac{\gamma^{2}}{2^{2}}\left(\tilde{T}_{k+2}^{N-2}-2 \tilde{T}_{k}^{N-2}+\tilde{T}_{k-2}^{N-2}\right)\right] .
$$

The $N-1$ above the equality sign means that only terms with range number $N-1$ are equal. When $k=1$,

$$
0 \stackrel{N-1}{=}\left[\tilde{T}_{0}^{1}, \tilde{T}_{1}^{N}+\frac{\gamma}{2} \tilde{T}_{2}^{N-1}+\frac{\gamma^{2}}{2^{2}}\left(\tilde{T}_{3}^{N-2}-\tilde{T}_{1}^{N-2}\right)\right] .
$$


For $k=N-4$ and $k=N-3$, we have

$$
0 \stackrel{N-1}{=}\left[\tilde{T}_{0}^{1}, \tilde{T}_{k}^{N}+\frac{\gamma}{2}\left(\tilde{T}_{k+1}^{N-1}-\tilde{T}_{k-1}^{N-1}\right)+\frac{\gamma^{2}}{2^{2}}\left(-2 \tilde{T}_{k}^{N-2}+\tilde{T}_{k-2}^{N-2}\right)\right],
$$

and finally, for $k=N-2$ and $k=N-1$,

$$
0 \stackrel{N-1}{=}\left[\tilde{T}_{0}^{1}, \tilde{T}_{k}^{N}+\frac{\gamma}{2}\left(\tilde{T}_{k+1}^{N-1}-\tilde{T}_{k-1}^{N-1}\right)+\frac{\gamma^{2}}{2^{2}} \tilde{T}_{k-2}^{N-2}\right] .
$$

We also have the relations

$$
0 \stackrel{N-1}{=}\left[\tilde{T}_{0}^{1}, \tilde{T}_{1}^{N}+\frac{\gamma}{2} \tilde{T}_{2}^{N-1}\right]
$$

and

$$
0=\left[\tilde{T}_{0}^{1}, \tilde{T}_{0}^{N}\right]+\left.\frac{\gamma}{2}\left[\tilde{T}_{0}^{1}, \tilde{T}_{1}^{N-1}\right]\right|_{N},
$$

where $\left.\right|_{N}$ on the right-hand side illustrates that the left-hand side is only equal to the term with range $N$ on the right-hand side. From this, we can deduce the following expression for the commuting charges ${ }^{1}$ :

$Q_{N}=\sum_{n=0}^{N-2}\left(\sum_{l=0 \vee W_{N, n}}^{\lfloor n / 2\rfloor} \frac{\gamma^{n}}{2^{n}} C_{n, l} \tilde{T}_{n-2 l}^{N-n}\right), \quad W_{N, n}= \begin{cases}-\frac{N-2 n-3}{2}, & N \text { odd } \\ -\frac{N-2 n-2}{2}, & N \text { even, }\end{cases}$

where the coefficients are given by (see appendix B)

$$
C_{n, l}=(-1)^{l}\left(\left(\begin{array}{l}
n \\
l
\end{array}\right)-\sum_{j=1}^{l}\left(\begin{array}{l}
n-j \\
l-j
\end{array}\right)\right)
$$

for $n>1$ and $C_{0,0}=1$ and $C_{1,0}=1$.

\section{Conclusion}

We have obtained an expression for a generic conserved charge of a local Hamiltonian satisfying the Temperley-Lieb algebra, in a simple form. This expression can be used to verify whether the generalization, which we have given here to the first few orders in $\lambda$, to the higher loop $N=4$ model, is integrable. We expect that when adding higher-loop contributions, the charges can also be expressed in a similar simple form. There are two separate questions we would like to have an answer to.

Firstly, is the Hamiltonian (13) integrable when including all orders in the parameter $\lambda$ ? And secondly, this Hamiltonian has the correct interaction range to given orders in $\lambda$ to describe a field theory, but will the dilatation operator of the $q$-deformed theory have the correct coefficients in front of the different interaction terms to be described by our model? We have seen that simply demanding the existence of one commuting charge puts strong conditions on the appearance of the field theory. It is enough for us to do a three-loop calculation on the field theory side to be able to rule out integrability if the coefficients do not come out as in equation (13) modulo lower charges and shift in $\lambda$. If the three-loop calculation would come out in favour of our model then it would be very interesting if someone could do a four-loop calculation.

$1\lfloor m\rfloor$ is the integer part of $m$, and $a \vee b:=\max (a, b)$. 
We would like to emphasize that the model is valid for any representation of the Temperley-Lieb algebra, and as such could very well describe the matrix of anomalous dimensions of some other field theory. Another interesting question is if these types of longrange spin chains always can be constructed as effective theories of Hubbard type, as is the case for the dilatation operator of $\mathcal{N}=4$ SYM.

To summarize, we have not yet revealed the infinite tower of commuting charges in the higher-loop deformed case, but we believe that not many days remain before they will be displayed.

\section{Acknowledgments}

We would like to thank Sergey Frolov and Matthias Staudacher for interesting discussions. This work was supported by the Alexander von Humboldt foundation.

\section{Appendix A. Some higher charges}

Here we have collected the five first of the two-loop charges:

$$
\begin{aligned}
Q_{1}= & \tilde{T}_{0}^{1}+\lambda \tilde{T}_{1}^{2} \\
Q_{2}= & \tilde{T}_{0}^{2}+\lambda \tilde{T}_{1}^{3} \\
Q_{3}= & \tilde{T}_{0}^{3}+\frac{\gamma}{2} \tilde{T}_{1}^{2}+\lambda\left(T_{1,0}^{4}+\gamma T_{2,0}^{3}-T_{1,1}^{3}-2 T B_{0,0}^{3}-\left(\frac{\gamma^{2}}{2}-4\right) T_{1,0}^{2}\right) \\
Q_{4}= & \tilde{T}_{0}^{4}+\frac{\gamma}{2} \tilde{T}_{1}^{3}+\lambda\left(T_{1,0}^{5}+\gamma T_{2,0}^{4}-T_{1,1}^{4}-2 T B_{0,0}^{4}-\left(\frac{\gamma^{2}}{2}-3\right) T_{1,0}^{3}\right) \\
Q_{5}= & \tilde{T}_{0}^{5}+\frac{\gamma}{2} \tilde{T}_{1}^{4}+\frac{\gamma^{2}}{2}\left(\tilde{T}_{2}^{3}+\tilde{T}_{0}^{3}\right)+\lambda\left(T_{1,0}^{6}+\gamma T_{2,0}^{5}-T_{1,1}^{5}+\frac{3 \gamma^{2}}{4} T_{3,0}^{4}-\gamma T_{2,1}^{4}+T_{1,2}^{4}\right. \\
& -2 T B_{0,0}^{5}-\gamma T B_{1,0}^{4}-2 T B_{0,0}^{3}+e_{\bar{i}, i+1, i+2, i+3}+e_{i, i+1, i+2, i+3} \\
& \left.+\frac{2}{\gamma}\left(-2+\frac{\gamma^{2}}{4}\right) T_{0,0}^{5}+\left(\frac{3 \gamma}{2}-\frac{3 \gamma^{3}}{8}\right) T_{2,0}^{3}+\left(3-\frac{3 \gamma^{2}}{4}+\frac{3 \gamma^{4}}{16}\right) T_{1,0}^{2}+\frac{3 \gamma^{2}}{4} T_{1,1}^{3}\right) .
\end{aligned}
$$

The charges are only specified modulo lower charges.

\section{Appendix B. The coefficients in the generic charge}

In figure B1, we have pictured Pascal's triangle together with the triangle of coefficients that we get for our charges. Our coefficient triangle can be obtained by adding up several of Pascal's triangles in such a way that the new triangle will have only zeroes on the right-hand side (in the picture we have not displayed the right-hand side of Pascal's triangle). As an example, we have marked the coefficient value 48 in our triangle. It can be obtained from Pascal's triangle by taking 84 minus the sum of the marked diagonal above 84 .

We can simply read off the values from the triangle when writing one of the charges, e.g. charge 8:

$Q_{8}=\tilde{T}_{0}^{8}+\frac{\gamma}{2} T_{1}^{7}+\frac{\gamma^{2}}{2^{2}}\left(T_{2}^{6}-T_{0}^{6}\right)+\frac{\gamma^{3}}{2^{3}}\left(T_{3}^{5}-2 T_{1}^{5}\right)+\frac{\gamma^{4}}{2^{4}}\left(-3 T_{2}^{4}+2 T_{0}^{4}\right)+\frac{\gamma^{5}}{2^{5}}\left(5 T_{1}^{3}\right)$. 


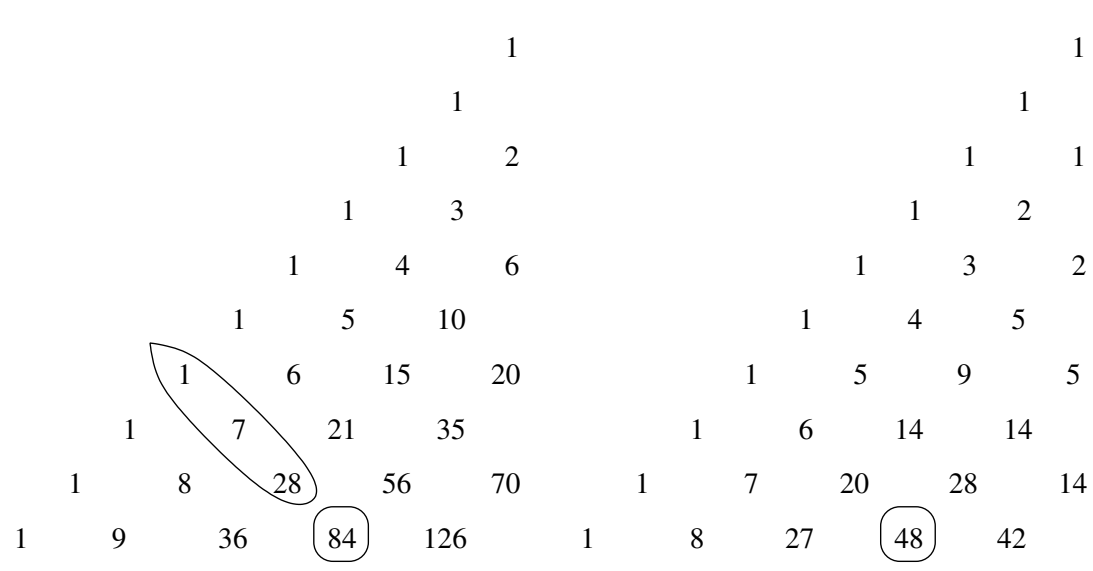

Figure B1. Pascal's triangle together with the coefficient triangle.

\section{References}

[1] Minahan J A and Zarembo K 2003 The Bethe-ansatz for $\mathcal{N}=4$ super Yang-Mills J. High Energy Phys. JHEP0303(2003)013 (Preprint hep-th/0212208)

[2] Beisert $\mathrm{N}$ and Staudacher M 2003 The $\mathcal{N}=4$ SYM integrable super spin chain Nucl. Phys. B $670439-63$ (Preprint hep-th/0307042)

[3] Beisert N, Kristjansen C and Staudacher M 2003 The dilatation operator of $\mathcal{N}=4$ conformal super Yang-Mills theory Nucl. Phys. B 664 131-84 (Preprint hep-th/0303060)

[4] Bern Z, Czakon M, Dixon L J, Kosower David A and Smirnov V A 2007 The four-loop planar amplitude and cusp anomalous dimension in maximally supersymmetric Yang-Mills theory Phys. Rev. D 75085010 (Preprint hep-th/0610248)

[5] Beisert N, McLoughlin T and Roiban R 2007 The f our-loop dressing phase of $\mathcal{N}=4$ SYM Phys. Rev. D 76046002 (Preprint hep-th/0705.0321)

[6] Serban D and Staudacher M 2004 Planar $\mathcal{N}=4$ gauge theory and the Inozemtsev long range spin chain J. High Energy Phys. JHEP06(2004)001 (Preprint hep-th/0401057)

[7] Inozemtsev V I 2003 Integrable Heisenberg-van Vleck chains with variable range exchange Phys. Part. Nucl. 34 166-93 (Preprint hep-th/0201001)

[8] Rej A, Serban D and Staudacher M 2006 Planar $\mathcal{N}=4$ gauge theory and the Hubbard model J. High Energy Phys. JHEP03(2006)018 (Preprint hep-th/0512077)

[9] Beisert N, Dippel V and Staudacher M 2004 A novel long range spin chain and planar $\mathcal{N}=4$ super Yang-Mills J. High Energy Phys. JHEP07(2004)075 (Preprint hep-th/0405001)

[10] Arutyunov G, Frolov S and Staudacher M 2004 Bethe ansatz for quantum strings J. High Energy Phys. JHEP10(2004)016 (Preprint hep-th/0406256)

[11] Beisert N and Staudacher M 2005 Long-range PSU $(2,2 \mid 4)$ Bethe ansatz for gauge theory and strings Nucl. Phys. B 727 1-62 (Preprint hep-th/0504190)

[12] Roiban R 2004 On spin chains and field theories J. High Energy Phys. JHEP09(2004)023 (Preprint hep-th/0312218)

[13] Berenstein D and Cherkis S A 2004 Deformations of $\mathcal{N}=4$ SYM and integrable spin chain models Nucl. Phys. B 702 49-85 (Preprint hep-th/0405215)

[14] Beisert N and Roiban R 2005 Beauty and the twist: the Bethe ansatz for twisted $\mathcal{N}=4$ SYM J. High Energy Phys. JHEP08(2005)039 (Preprint hep-th/0505187)

[15] Freyhult L, Kristjansen C and Månsson T 2005 Integrable spin chains with $U(1)^{3}$ symmetry and generalized Lunin-maldacena backgrounds J. High Energy Phys. JHEP12(2005)008 (Preprint hep-th/0510221)

[16] Bundzik D and Månsson T 2006 The general Leigh-Strassler deformation and integrability J. High Energy Phys. JHEP01(2006)116 (Preprint hep-th/0512093)

[17] Månson T 2007 The Leigh-Strassler deformation and the quest for integrability J. High Energy Phys. JHEP06(2007)010 (Preprint hep-th/0703150) 
[18] Frolov S A, Roiban R and Tseytlin A A 2005 Gauge-string duality for superconformal deformations of $\mathcal{N}=4$ super Yang-Mills theory J. High Energy Phys. JHEP07(2005)045 (Preprint hep-th/0503192)

[19] Leigh R G and Strassler M J 1995 Exactly marginal operators and duality in four-dimensional $\mathcal{N}=1$ supersymmetric gauge theory Nucl. Phys. B 447 95-136 (Preprint hep-th/9503121)

[20] Ikhlef Y, Jacobsen J L and Saleur H 2006 A staggered six-vertex model with non-compact continuum limit (Preprint cond-mat/0612037)

[21] Grabowski M P and Mathieu P 1994 Quantum integrals of motion for the Heisenberg spin chain (Preprint hep-th/9403149)

[22] Anshelevich V V 1980 First integrals and stationary states for quantum Heisenberg spin dynamics Theor. Math. Phys. 43 350-2

[23] Grabowski M P and Mathieu P 1995 Structure of the conservation laws in integrable spin chains with short range interactions Ann. Phys. 243 299-371 (Preprint hep-th/9411045)

[24] Tetelman M G 1982 Lorentz group for two-dimensional integrable lattice systems Sov. Phys._JETP 55 306-10 